\title{
Dietary Fat Composition Influences Tissue Lipid Profile and Gene Expression in Fischer-344 Rats
}

\author{
Albert L. Zhou • Korry J. Hintze • \\ Rafael Jimenez-Flores · Robert E. Ward
}

\begin{abstract}
The AIN-76A diet causes fatty liver in rodents when fed for long periods of time. The aim of this study was to utilize fatty acid analysis and transcriptomics to investigate the effects of different fat sources in the AIN-76A diet on tissue lipid profiles and gene expression in male, weanling Fischer-344 rats. Animals were fed isocaloric diets that differed only in the fat source: (1) corn oil (CO) (2) anhydrous milk fat (AMF), and (3) AMF supplemented with $10 \%$ phospholipids from the milk fat globule membrane (AMFMFGM). There were no differences in food intake, body weight, growth rate, or body fat composition among the groups, and the fatty acid compositions of red blood cells (RBC), plasma, muscle, and visceral adipose tissues reflected the dietary fat sources. Modifying the fat source resulted in 293 genes differentially regulated in skeletal muscle, 1,124 in adipose, and 831 in liver as determined by analysis of variance (ANOVA). Although tissue fatty acid profiles mostly reflected the diet, there were several quantitative differences in lipid classes in the liver and plasma. The AMF diet resulted in the highest level of hepatic triacylglycerols, but the lowest level in plasma. The CO diet resulted in significant accumulation of hepatic unesterified fatty acids and decreased DGAT expression and activity, a potential trigger for steatohepatitis. These results indicate that the fatty acid composition and presence of polar lipids in the AIN-76A diets
\end{abstract} have significant effects on lipid partitioning, gene expression,

and potentially the development of liver pathology.

$\begin{array}{ll}\text { Abbreviations } \\ \text { ACF } & \text { Aberrant crypt foci } \\ \text { AMF } & \text { Anhydrous milk fat } \\ \text { ANOVA } & \text { Analysis of variance } \\ \text { BHT } & \text { Butylated hydroxytoluene } \\ \text { CE } & \text { Cholesteryl esters } \\ \text { CHD } & \text { Coronary heart disease } \\ \text { CO } & \text { Corn oil } \\ \text { FAME } & \text { Fatty acid methyl ester(s) } \\ \text { FFA } & \text { Unesterified fatty acids } \\ \text { HUFA } & \text { Highly unsaturated fatty acid(s) } \\ \text { MFGM } & \text { Milk fat globule membrane } \\ \text { NAFLD } & \text { Nonalcoholic fatty liver disease } \\ \text { NASH } & \text { Nonalcoholic steatohepatitis } \\ \text { PL } & \text { Phospholipid(s) } \\ \text { PUFA } & \text { Polyunsaturated fatty acids } \\ \text { RBC } & \text { Red blood cell(s) } \\ \text { TAG } & \text { Triacylglycerol(s) } \\ \text { TLC } & \text { Thin-layer chromatography }\end{array}$

\section{Introduction}

The AIN-76 diet is a purified rodent diet that was developed in the late 1970s by an ad hoc committee formed by 
the American Institute of Nutrition (AIN) [1]. One rationale for developing this diet was to provide researchers with a nutritionally adequate diet that would allow for standardizing studies between laboratories. Not long after this diet was developed several groups reported undesirable physiological effects linked to the diet, such as hemorrhagic deaths [2], nephrocalcinosis [3], and fatty liver [4]. An amended formulation of the diet (AIN-76A) was developed in 1980 with an increased vitamin $\mathrm{K}$ content to address the hemorrhagic effect [5]. In 1993 two revised diets were formulated to replace the AIN-76A, a version for growing animals (AIN-93G) and a maintenance formula for mature animals (AIN-93M). These revised diets contained a higher calcium to phosphorus ratio (from 0.75 to 1.3) to prevent the nephrocalcinosis found in female rats consuming the AIN-76A diet [1]. In addition, the carbohydrate composition was changed from $50 \%$ sucrose and $15 \%$ cornstarch in the AIN-76A formula to $\sim 40 \%$ cornstarch, $15 \%$ dextrinized cornstarch, and $10 \%$ sucrose in the AIN-93G diet. This change represents a fivefold decrease in the fructose content of the diet and is not associated with the accumulation of hepatic lipids caused by long-term feeding of the high sucrose levels [6]. Despite the availability of the improved rodent diets, the AIN-76A still remains popular in specific research fields, such as in studies investigating the effects of diet on colon cancer using the aberrant crypt foci model (ACF). In fact, in a database maintained to compare the efficacy of diet modulation on the incidence of ACF, the overwhelming majority of studies have utilized the AIN-76A [7].

The propensity of the AIN-76A diet to result in accumulation of hepatic lipids is potentially of interest to human nutrition, as it is estimated that between 20 and $30 \%$ of adults in Western countries have nonalcoholic fatty liver disease (NAFLD) and this level rises to between 70 and $90 \%$ in obese individuals and those with diabetes [8]. To test the hypothesis that high levels of dietary fructose are responsible for the accumulation of hepatic lipids, Bacon et al. [9] tested the effects of modifying the sucrose content of the AIN-76A diet in a 3-week feeding study in rats. According to the results, diets containing greater than $25 \%$ sucrose resulted in significantly higher hepatic triacylglycerols (TAG) than ones with less than $20 \%$ sucrose or chow.

We recently conducted an ACF study utilizing the AIN$76 \mathrm{~A}$ diet to investigate the potential chemopreventive effects of a complex milk fat fraction [10]. In the study we used three diets with different fat compositions: (1) corn oil (CO), (2) anhydrous milk fat (AMF), and (3) anhydrous milk fat supplemented with milk fat globule membrane (AMF-MFGM). CO diet is the standard fat source for the AIN-76A diet, and for the AMF diet, the $5 \%$ mass of fat was replaced with AMF. Formulation of the AMF-MFGM diet was achieved by utilizing an isolate of MFGM (providing $10 \%$ phospholipids of total fat) that was isolated from cream, and which also contained protein, carbohydrate, and minerals in addition to the fat. The details of the diet formulation have been reported previously [10].

MFGM is derived from the apical surface of mammary epithelial cells and surrounds the fat droplets in milk [11]. It is composed primarily of TAG, proteins, and phospholipids (PL) [12]. The composition of the polar lipids of MFGM can vary according to the method of isolation, but the major constituents are phosphatidylcholine $(\sim 32 \%)$, phosphatidylethanolamine $(32 \%)$, sphingomyelin $(\sim 24 \%)$, phosphatidylinositol $(\sim 4 \%)$, phosphatidylserine $(\sim 3 \%)$, and gangliosides $(\sim 3 \%)$ [13]. A large amount of this material is produced in the USA each year as a byproduct of butter production, and is available as a food ingredient. Owing to its unique lipid profile and membrane protein profile, MFGM has been suggested as a potentially bioactive food ingredient with novel nutritional functionalities [14]. Despite this supposition, few studies have been conducted in vivo with either animal models or humans to evaluate the potential of this material as a bioactive ingredient.

The goal of this study was to investigate lipid metabolism in rats fed a diet known to induce hepatic stress. Our approach involved subjecting lipid-metabolizing tissues to two different, yet comprehensive analytical techniques (lipid and gene expression profiling) to understand how the dietary fats affected the lipid metabolism.

\section{Materials and Methods}

Animals and Diets

Eleven animals used in this study were among a group of 63 male, weanling Fischer-344 rats (Charles River Laboratories) used in another study [10]. They were randomly assigned to one of three isocaloric dietary treatments that differed only in the fat source [10]. After a 7-day acclimation period on standard chow diets, the rats were individually housed in a room controlled for temperature, humidity, and light cycle and were given free access to experimental diets and deionized water. Food intake and weight were measured weekly. All experimental protocols involving animals were approved by the Utah State University Institutional Animal Care and Use Committee.

Animals were fed experimental diets for 12 weeks. After MRI analysis of body composition (EchoMRI$900^{\mathrm{TM}}$ ), rats were killed by cardiac puncture following ketamine/xylazine anesthesia. Liver, adipose, and skeletal muscle tissues and blood samples were collected, flash frozen in liquid nitrogen, and stored at $-80{ }^{\circ} \mathrm{C}$ for until the time of analysis. 


\section{Lipid Extraction}

Tissue samples were removed from the freezer and a sample of tissue was cut into small pieces with a razor and placed in a mortar with liquid nitrogen and ground to obtain a fine homogenous powder. About $200 \mathrm{mg}$ of the tissue powder or plasma from each sample was weighed and put into a screw-capped glass tube. Surrogate standards, including sphingomyelin, phosphatidylcholine, phosphatidylserine, phosphatidylethanolamine, diacylglycerol (DG), unesterified fatty acids (FFA), TAG, and cholesteryl esters (CE), were prepared in chloroform. Surrogate standards $(100 \mu \mathrm{l})$ for different lipid classes were added to each sample and weights were recorded. Lipids were extracted by the method of Folch et al. [15] with slight modifications. Samples were mixed with $5 \mathrm{ml}$ chloroform/methanol (2:1 with butylated hydroxytoluene, BHT). The whole mixture was sonicated and then agitated for 15-20 min in an orbital shaker at room temperature. The mixture was washed with 0.2 volumes $(1 \mathrm{ml}$ for $5 \mathrm{ml}$ solvent mixture) of $0.9 \% \mathrm{NaCl}$ solution and vortexed for $20 \mathrm{~s}$. Subsequently, the mixture was centrifuged at $1,500 \times g$ for $10 \mathrm{~min}$ to separate the two phases. The lower (chloroform) phase containing lipids was collected and evaporated under a nitrogen stream and then reconstituted in a small volume of hexane with BHT in a 4-ml amber vial and stored at $-80{ }^{\circ} \mathrm{C}$ until further analysis.

\section{Separation and Recovery of Different Lipid Classes}

Individual lipid classes of the extracted lipid were separated using thin-layer chromatography (TLC). Extracted lipid from each tissue was diluted by hexane with BHT such that $20 \mu \mathrm{l}$ of solution contained around $2.5 \mathrm{mg}$ of lipid. Aliquots of $20 \mu \mathrm{l}$ were spotted on a $20 \times 20 \mathrm{~cm}$ silica gel $60 \AA$ analytical plate (500 $\mu \mathrm{m}$ layer) (Whatman Inc., Florham Park, NJ). Lipid class standards were also spotted for detecting target bands. Total lipid classes were separated by developing the plate in a solvent system containing hexane, diethyl ether, and formic acid in the ratio of $80: 20: 2(\mathrm{v} / \mathrm{v} / \mathrm{v})$. The TLC plate was then sprayed with $0.05 \%$ primulin in acetone/water $(8: 2 \mathrm{v} / \mathrm{v})$. Individual lipid bands on the TLC plate were detected under a handheld UV lamp and the margins were marked with a pencil. Target lipid classes bands were scraped from the TLC plate using a small razor blade and collected into screw-capped glass tubes. Recovered lipid classes from total lipid TLC plate were PL, DG, FFA, TAG, and CE.

\section{Fatty Acid Methyl Esters}

Recovered lipid classes were transesterified into fatty acid methyl esters (FAME) using the method of Curtis et al.
[16] with slight modifications. In each tube, $0.8 \mathrm{ml}$ hexane and $1.2 \mathrm{ml} 10 \%(\mathrm{v} / \mathrm{v})$ acetyl chloride in methanol were added. Tubes were capped, vortexed for $20 \mathrm{~s}$, and placed in an oven at $100{ }^{\circ} \mathrm{C}$ for $40 \mathrm{~min}$. Upon completion of the incubation period, samples were removed and allowed to cool to room temperature. Then $2 \mathrm{ml}$ of a $6 \%$ sodium carbonate solution and $0.4 \mathrm{ml}$ hexane were added into each tube. On subsequent vortexing and centrifuging of tubes two distinct phases were obtained. The top organic layer was removed and transferred to gas chromatography (GC) vials. Solvent was evaporated under a nitrogen stream and FAME were collected in $100 \mu$ l of hexane with BHT and transferred to GC vial inserts. The samples were subsequently analyzed by GC using a GC2010 (Shimadzu Scientific Instruments, Columbia, MD).

\section{GC Data Analysis}

For each GC run, standard curves were generated using commercially available FAME standards (Nu-Chek Prep, Elysian, MN). To establish the linearity of the detector response, a three-point calibration was generated with every sample set. The calibration standard, GLC-463, contained 42 fatty acids representing most of the common species found in mammalian tissues and dairy products. Chromatograms of sample FAME were compared with those of FAME standards to identify target fatty acids. The peak area for each fatty acid was then normalized to the peak area and concentration of the corresponding surrogate standard. Next, the molar concentration of the analytes was calculated and then converted to moles of the corresponding lipid classes through molecular weight calculations. The molar concentration of lipid classes was normalized to tissue sample weights to obtain concentrations ( $\mu \mathrm{mol} / \mathrm{g}$ tissue) in each tissue. Lastly, the mole percentage of each fatty acid species was calculated.

\section{Gene Expression Analysis}

Total RNA was extracted from liver, muscle, and adipose tissue and prepared as previously described [10]. Microarray data were generated by Genome Quebec using the Illumina platform (Illumina, Inc., San Diego, CA). Data were analyzed using FlexArray 1.6, a custom statistical software program developed by Genome Quebec [17]. The expression data were filtered to remove feature ids that have not been detected. Next, a background adjustment and normalization were carried out using the Lumi algorithm (based on lumi Bioconductor package) in FlexArray 1.6. Background correction was carried out by using negative controls. Variance was stabilized by the variance-stabilizing transformation (VST) method and subsequently a robust spline normalization was conducted. Analysis of 
variance (ANOVA) was conducted to evaluate significant diet effects on gene expression across treatment groups. The Cyber-T algorithm was employed to identify differentially regulated genes (CT gene lists) among tissues between treatment groups from ANOVA gene lists [18] followed by the Benjamini-Hochberg false discovery rate multiple testing correction [19]. The CT gene lists were then subjected to Kyoto Encyclopedia of Genes and Genomes (KEGG) pathway analysis using ArrayTrack v.3.5.0 (NCTR/FDA) [20].

\section{Real-Time PCR}

Total RNA was extracted from liver samples using the RNAeasy Mini-Kit (Qiagen, Valencia, CA). RNA quality was checked and the concentration measured by NanoDrop 1000 (Thermo Fisher, Waltham, MA). RNA (1 $\mu \mathrm{g})$ was converted to cDNA using high-capacity cDNA reverse transcription kit (Life Technologies, Carlsbad, CA). Validated and predesigned TaqMan primers and probes (Life Technologies) were used to quantify cytochrome P450, family 8, subfamily b, polypeptide 1 (Cyp8b1, ID: Rn00579921_s1), 1-acylglycerol-3-phosphate $O$-acyltransferase 2 (Agpat2, ID: Rn01438505_m1), and angiopoietinlike 4 (Angptl4, ID: Rn01528817_m1). Beta-actin (Actb, ID: ID Rn00667869_m1) was used as an endogenous control. Real-time PCR amplifications were carried out in a DNA Engine Opticon ${ }^{\circledR} 2$ two-color real-time PCR detection system (Biorad, Hercules, CA). PCR results were analyzed with the Opticon Monitor 3 software (Biorad). The comparative $C_{\mathrm{t}}$ method was used to quantify gene expression. $\Delta C_{\mathrm{t}}$ was obtained by normalizing to beta-actin. $\Delta \Delta C_{\mathrm{t}}$ was determined by the arithmetic formula described by López-Parra et al. [21]. Symmetrical raw fold change was obtained by comparing $\Delta \Delta C_{\mathrm{t}}$ values of two groups compared.

\section{Liver Fat Analysis by Histology}

Cryostat sections in $6 \mu \mathrm{m}$ thickness were made from liver samples taken out of $-80{ }^{\circ} \mathrm{C}$ freezer. The tissue sections were brought to room temperature on glass slides. The slides were dipped a few times in $60 \%$ triethyl phosphate and then stained in $0.5 \%$ Oil Red O (in $60 \%$ triethyl phosphate) for $15 \mathrm{~min}$. After being rinsed in water for $2 \mathrm{~min}$, the slides were counterstained in Harris modified method hematoxylin stain (Thermo Fisher, Waltham, MA) for $2 \mathrm{~min}$. The slides were placed in saturated lithium carbonate solution for $10 \mathrm{~s}$, rinsed in water for $5 \mathrm{~min}$, and held in water. The slides were mounted with warm glycerin jelly and observed with light microscopy. Color images were captured with a digital camera attached to the microscope. Lipids in randomly sampled areas from each slide were quantified by ImageJ [22]. The fat content of each sample was expressed by quantification of Oil Red $\mathrm{O}$-stained areas as a percentage of whole area.

Liver Diacylglycerol Acyltransferase (DGAT) Enzyme Assay

The microsomal fraction was obtained by the methods of Ko et al. [23] and Coleman [24]. Rat liver (approximately $1.5 \mathrm{~g}$ ) was minced and then homogenized in $15 \mathrm{ml}$ of STE buffer (0.25 M sucrose, 10 mM Tris-HCl, pH 7.4, 1.0 mM EDTA) with a Teflon-glass homogenizer at medium speed. The homogenate was centrifuged at $14,000 \times g$ for $20 \mathrm{~min}$ at $4{ }^{\circ} \mathrm{C}$. The supernatant was centrifuged at $100,000 \times g$ for $1 \mathrm{~h}$ at $4{ }^{\circ} \mathrm{C}$ to obtain a microsomal pellet. The pellet was suspended in STE buffer without EDTA and centrifuged at $100,000 \times g$ for $1 \mathrm{~h}$ at $4{ }^{\circ} \mathrm{C}$. The final pellet was resuspended in STE buffer without EDTA. Total protein in final solution was quantified by the Bradford assay. The microsomal fractions were stored in aliquots at $-80{ }^{\circ} \mathrm{C}$.

A fluorescent DGAT assay was carried out as described by McFie and Stone [25]. Briefly, a master mix was prepared in a test tube containing $20 \mu \mathrm{l}$ of $1 \mathrm{M}$ Tris- $\mathrm{HCl}(\mathrm{pH}$ 7.6), $4 \mu \mathrm{l}$ of $1 \mathrm{M} \mathrm{MgCl}, 10 \mu \mathrm{l}$ of $4 \mathrm{mM}$ dioleoyl-snglycerol, $10 \mu \mathrm{l}$ of $12.5 \mathrm{mg} / \mathrm{ml}$ bovine serum albumin, $10 \mu \mathrm{l}$ of $500 \mu \mathrm{M}$ NBD-palmitoyl CoA, and $96 \mu \mathrm{l}$ of water per reaction. Tubes were pre-incubated in a $37{ }^{\circ} \mathrm{C}$ water bath for $2 \mathrm{~min}$ and $50 \mu \mathrm{l}$ of protein sample was added to start the reaction, which was held at $37^{\circ} \mathrm{C}$ for $10 \mathrm{~min}$ with occasional shaking. The reaction was terminated by addition of $4 \mathrm{ml} \mathrm{CHCl}_{3} / \mathrm{MeOH}$ (2:1, v/v). Samples were added to $800 \mu \mathrm{l}$ of water and allowed to sit at room temperature for $1 \mathrm{~h}$. Tubes were centrifuged at $3,000 \times g$ for $5 \mathrm{~min}$ to separate aqueous and organic phases. The organic phase was removed via pipette and dried under a stream of nitrogen. Lipids were resuspended in $50 \mu \mathrm{CHCl}_{3} / \mathrm{MeOH}$ (2:1) and spotted on a $20 \times 20 \mathrm{~cm}$ TLC plate. TLC plates were developed in the solvent system, hexane/diethyl ether/ acetic acid (80:20:1, v/v/v). The plates were air-dried for $1 \mathrm{~h}$ and scanned by Typhoon Trio + Laser Imager 7 (GE Healthcare, Waukesha, WI). The following settings were used: excitation, blue (488 nm) LED laser light source; emission, $520 \mathrm{~nm}$ BP emission filter. Fluorescence was quantified by ImageJ [22]. The newly synthesized NBDTAG was quantified as units (fluorescence intensity) of NBD-TAG formed per minute per milligram of protein.

Statistical Analysis

A one-way ANOVA was performed using SAS software version 9.2 (SAS Institute Inc.) to perform comparisons among groups or using FlexArray 1.6 for gene expression among groups and tissues. Group means were compared 
using Cyber-T algorithm for gene expression data and Ryan-Einot-Gabriel-Welsch multiple range test for other data. Data were reported as mean \pm standard deviation.

\section{Results}

Fatty Acid Profile of Diets

The most abundant fatty acids of the test diets are shown in Fig. 1. Both the milk-fat-based diets have similar fatty acid profiles, and are very different from the $\mathrm{CO}$ diet. The milk fat diets both have significant contributions of saturated fatty acids $(\sim 65 \%)$, whereas in the $\mathrm{CO}$ diet the percentage is much lower $(\sim 17.5 \%)$. All diets have similar levels of monounsaturates, with oleic acid contributing approximately $25 \%$. The other notable difference is in the polyunsaturated fatty acid content (PUFA) content (CO $54 \%$, AMF $4.5 \%$, and AMF-MFGM $6 \%$ ) and n-6/n-3 ratio (CO 54:1, AMF 6:1, AMF-MFGM 7:1).

Food Intake, Body Weight, Growth Rate, and Body Fat Composition

There were no significant differences in terms of food intake, final body weight, growth rate, or body fat composition (MRI analysis) among groups [10].

Fatty Acids Profile of Red Blood Cells

The red blood cell (RBC) fatty acid profile was measured to compare the $\mathrm{CO}$ diet to the milk-fat-based diets, and to determine if the higher polar lipid content of the AMF-MFGM had an effect compared with the AMF diet. RBC fatty acids that were significantly affected by the diets and present at $>1 \%$ of total fatty acids are shown in Fig. 2. Not surprisingly,

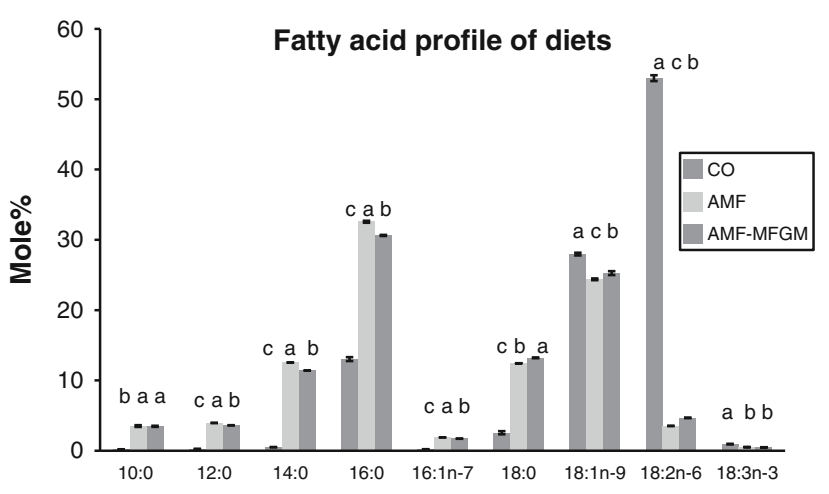

Fig. 1 Fatty acid profile of experimental diets. Only fatty acids significantly different across groups and present at $>1 \%$ of total fatty acids are shown. Means in a row with different superscripts are significantly different $(p<0.05)$

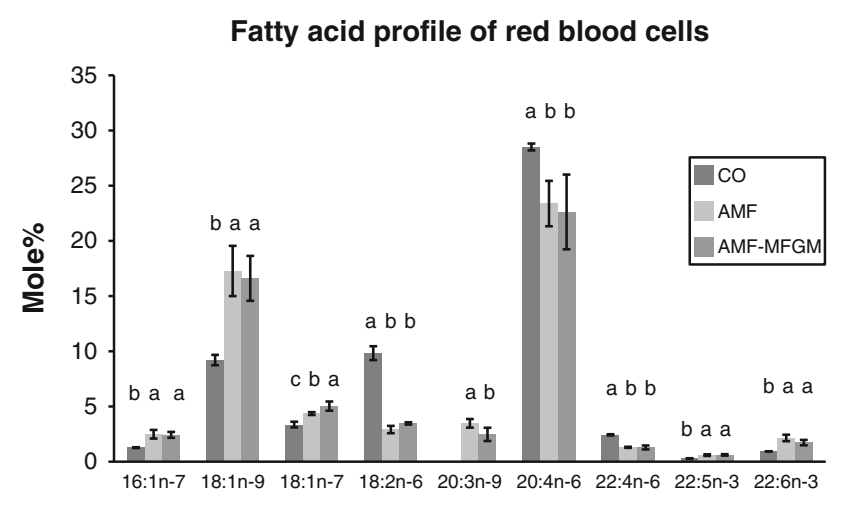

Fig. 2 Red blood cell (RBC) fatty acids from rats fed CO (corn oil), AMF (anhydrous milk fat), and AMF-MFGM (anhydrous milk fatmilk fat globule membrane). Only fatty acids significantly different across diet groups and present at $>1 \%$ of total fatty acids are shown. Means in a row with different superscripts are significantly different $(p<0.05)$

the $\mathrm{CO}$ cohort had a higher percentage of $18: 2 \mathrm{n}-6,20: 4 \mathrm{n}-6$, and $22: 4 n-6$, all n-6 fatty acids, presumably because of the high linoleic acid content of the diet. In addition, these animals had lower percentages of $16: 1 \mathrm{n}-7,18: 1 \mathrm{n}-9,18: 1 \mathrm{n}-7,20: 3 \mathrm{n}-9$, 20:5n-3, 22:5n-3, and 22:6n-3 compared to the animals fed the milk fat diets. The RBC from animals fed the two milk-fatbased diets had remarkably similar fatty acid profiles with two statistically relevant differences. Namely, the AMF-MFGMfed animals had a higher percentage of 18:1n-7 and a lower percentage of 20:3n-9.

Fatty Acids Profile of Skeletal Muscle Tissue and Visceral Adipose Tissue

As with the RBC, there were large differences between the fatty acid profile in skeletal muscle and visceral adipose tissue of the rats fed the $\mathrm{CO}$ diet versus the milk fat diets which were virtually identical. In skeletal muscle, the COfed animals had significantly less 16:1n-7, 18:1n-9, and 20:3n9, and more 18:2n-6, 20:4n-6, and 22:4n-6 (Fig. 3). Unlike skeletal muscle, the contribution of long chain fatty acids to the adipose lipids was low (Fig. 4). However, among the major fatty acids ( $>1 \%$ total), the $\mathrm{CO}$ animals had less 16:0, 16:1n-7, 18:0, and 18:1n-9 and more 18:2n6 . The only fatty acid that differed among the animals fed the milk fat diets was 20:3n9, which in both tissues was slightly lower in the AMF-MFGM group.

\section{Fatty Acids Profile of Liver Tissue}

Fatty acids in liver tissue which were significantly affected by the $\mathrm{CO}$, AMF, and AMF-MFGM diets and which contribute $>1 \%$ of fatty acids in one tissue are presented in Fig. 5. Animals from the CO group had less 16:1n-7, 18:1n-9, 20:3n9, and 22:6n-3 and more 18:2n-6 and 20:4n-6. Unlike the 


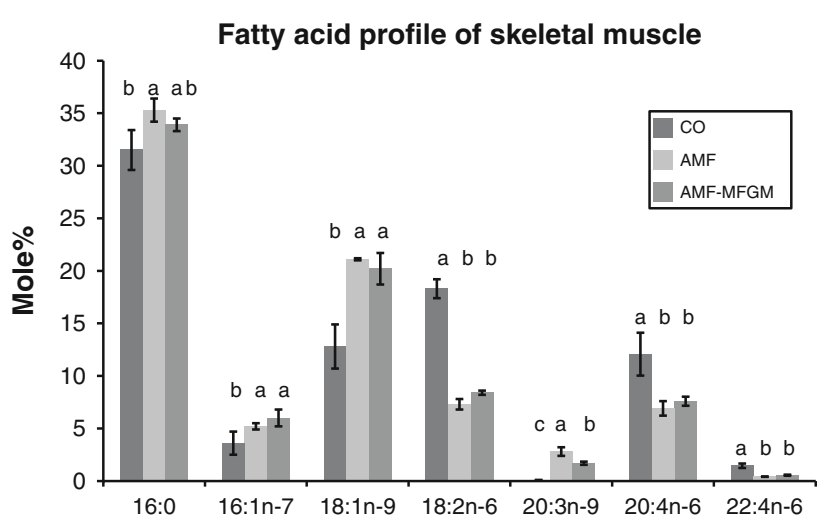

Fig. 3 Fatty acid profile of skeletal muscle from rats fed CO (corn oil), AMF (anhydrous milk fat), and AMF-MFGM (anhydrous milk fat-milk fat globule membrane). Only fatty acids significantly different across diet groups and present at $>1 \%$ of total fatty acids are shown. Means in a row with different superscripts are significantly different $(p<0.05)$

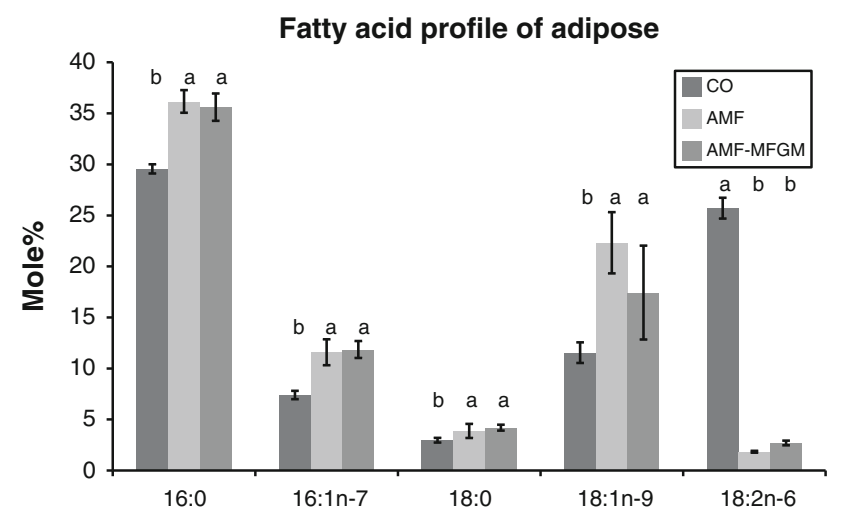

Fig. 4 Fatty acid profile of visceral adipose tissue from rats fed $\mathrm{CO}$ (corn oil), AMF (anhydrous milk fat), and AMF-MFGM (anhydrous milk fat-milk fat globule membrane). Only fatty acids significantly different across diet groups and present at $>1 \%$ of total fatty acids are shown. Means in a row with different superscripts are significantly different $(p<0.05)$

RBC, skeletal muscle, and adipose tissues, supplementation of the AMF diet with MFGM results in several differences in the fatty acid profile of the liver. For example, of the six fatty acids which are significantly affected across the diets and present at $>1 \%$, five are significantly different in livers of the animals fed the AMF and AMF-MFGM diets. Thus, the increased polar lipid concentration of the AMF-MFGM diet appears to have significant effects on liver lipid metabolism and partitioning. The largest differences are in 18:1n-9, which is higher in the AMF animals, and 20:4n-6 and 22:6n-3 which are higher in the AMF-MFGM cohort.

\section{Plasma, Visceral Adipose, and Liver Lipid Classes}

To better understand the differences in lipid partitioning as affected by the CO, AMF, and AMF-MFGM diets, plasma

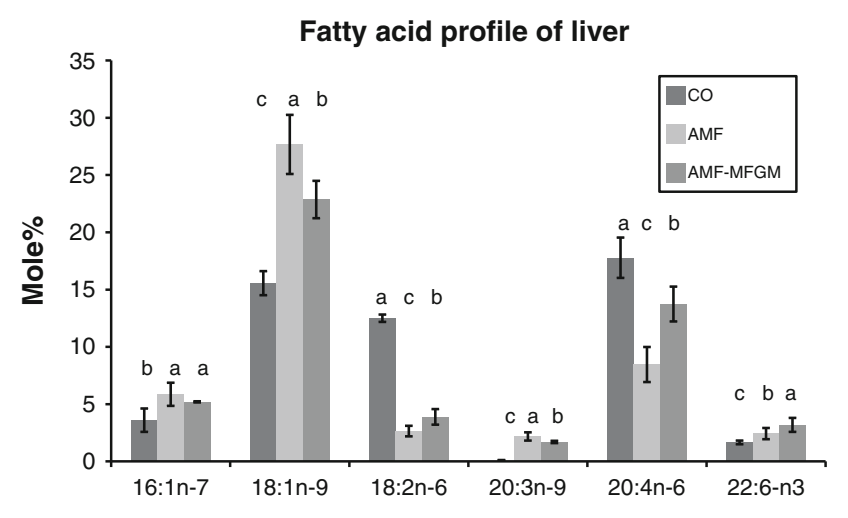

Fig. 5 Fatty acid profile of liver tissue from rats fed CO (corn oil), AMF (anhydrous milk fat), and AMF-MFGM (anhydrous milk fatmilk fat globule membrane). Only fatty acids significantly different across diet groups and present at $>1 \%$ of total fatty acids are shown. Means in a row with different superscripts are significantly different $(p<0.05)$

adipose and liver lipid classes were analyzed and classes with significant differences are shown in Fig. 6. Interestingly, the animals consuming AMF as the fat source had less total PL in plasma than animals fed the CO and AMFMFGM diets. Additionally, there was less TAG than the animals fed the AMF-MFGM diet. In the visceral adipose, there was more TAG in the animals fed $\mathrm{CO}$ than those fed the AMF-MFGM. In the liver, there was more FFA in the animals fed the $\mathrm{CO}$ diet than in those fed the AMF-MFGM diet, and a similar trend for the AMF animals, although this was not significant. On the other hand, the $\mathrm{CO}$ diet resulted in less accumulated TAG than the AMF diet, with the AMF-MFGM diet in between. Lastly, both the $\mathrm{CO}$ and the AMF-MFGM diets resulted in lower hepatic CE than did the AMF diet.

\section{Tissue Gene Expression}

In total, 293, 1,124, and 831 genes were found to be differentially expressed respectively in skeletal muscle, adipose, and liver at $p<0.05$. To identify metabolic pathways that were affected across the three diets all the genes that were differentially regulated according to the ANOVA were analyzed with the program ArrayTrack, a free software tool developed by the National Center for Toxicological Research (NCTR) and the US Food and Drug Administration (FDA). All three diets were compared and the data generated from this analysis are summarized in Table 1. On a diet comparison basis, the fewest number of pathways affected across the three tissues were between the two milk-fat-fed animal groups (22 pathways) and the most pathways were affected between the CO and AMF-MFGM groups (40). In specific tissues, there were 18 pathways differentially affected in skeletal muscle, 31 in adipose, and 
Fig. 6 Quantitative analysis of lipid classes from plasma, visceral adipose, and liver. $P L$ phospholipids, $T A G$ triacylglycerols, FFA free fatty acids, $C E$ cholesteryl esters. Means in a row with different superscripts are significantly different $(p<0.05)$
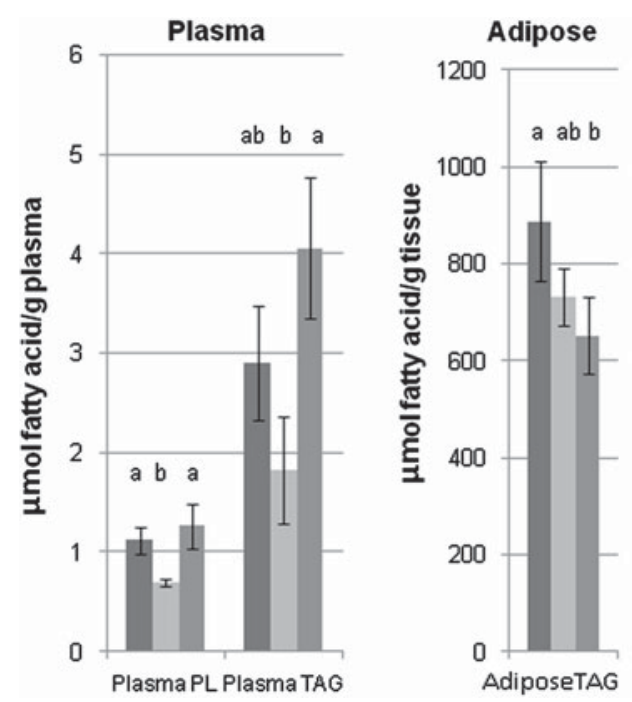

40 in the liver. In specific diet/tissue comparisons, the fewest number of pathways were affected in skeletal muscle between the $\mathrm{CO}$ and the AMF diets (2). There were 16 pathways affected between the AMF and $\mathrm{CO}$ diets in the liver and 16 pathways between the AMFMFGM and CO diets in adipose. In the lower half of Table 1 the pathways that were significantly affected across at least two diet combinations are shown. For example, in the muscle tissue, the circadian rhythm pathway (KEGG rno 04710) was significantly affected between all three diets, whereas adipocytokine signaling (rno00020) was only affected in the diet combinations in which AMF was compared. Similar to the fatty acid data of the selected tissues, there were fewer pathways affected in the muscle and adipose tissue compared to the liver. A striking feature of the pathway analysis shown in the bottom half of Table 1 is that only three pathways were different in the liver between the two milk fat diets, whereas $>10$ were affected when these diets were compared to the $\mathrm{CO}$ diet.

Although the two milk fat diets were nearly identical at the fatty acid level, there were several differences in the liver and plasma lipid profiles of the rats fed the AMF and AMF-MFGM diets. Therefore, the expression of several genes of interest was determined using RTPCR and the results are shown in Table 2. The three genes selected were chosen from the KEGG pathways differentially affected by the milk fat diets in Table 1 . The results of the RT-PCR in Table 2 are expressed relative to $\beta$-actin, and thus a smaller number indicates a higher expression level. Comparing the two milk fat groups, all three genes are more highly expressed in the livers of the AMF-MFGM rats compared to the AMF group.
Liver Fat Analysis by Histology and DGAT Assay

In general, both the lipid analysis and the gene expression profiling indicated that the diets had the greatest effect on the liver. Although there was not a quantitative difference in the fatty acid content of the livers, there were significant differences in distributions across lipid classes (Fig. 6). For example, there is more triacylglycerol in the livers of rats fed AMF compared to the CO, yet the total fatty acid was not different. Interestingly, expression of diacylglycerol $O$-acylransferase 2 (DGAT2), was lower in the CO-fed animals than in the other two groups. Consequently, the livers were stained with Oil Red O and DGAT activity was measured via enzymatic assay of the microsomal fraction, and the results are shown in Fig. 7. There was significantly less staining of the hepatic tissue of the CO-fed rat (Fig. 7a), and this corresponded with lower DGAT activity (Fig. 7b).

\section{Discussion}

The main focus of this work was to utilize tissue fatty acid profiling and transcriptomics to determine the effects of changing the lipid source of the AIN-76A diet on lipid metabolism. This work was conducted on key tissues involved in lipid metabolism, storage, and processing (skeletal muscle, visceral adipose, and liver) as well as plasma. The fat sources used in formulating the diets (corn oil, anhydrous milk fat, and anhydrous milk fat supplemented with milk fat globule membrane) were originally selected for an ACF study previously reported [10]. In that study, as in most ACF studies, the CO diet was selected as a control, whereas the AMF-MFGM diet was included to 
Table 1 Metabolic pathways significantly affected across all three diet combinations

\begin{tabular}{|c|c|c|c|c|c|}
\hline \multirow[t]{3}{*}{ Tissue } & \multicolumn{5}{|l|}{ Diets } \\
\hline & \multicolumn{5}{|l|}{ Number of pathways affected } \\
\hline & AMF versus $\mathrm{CO}$ & AMF-MFGM versus $\mathrm{CO}$ & \multicolumn{2}{|c|}{ AMF-MFGM versus AMF } & All \\
\hline Muscle & 2 & 11 & \multicolumn{2}{|l|}{5} & 18 \\
\hline Adipose & 9 & 16 & \multicolumn{2}{|l|}{6} & 31 \\
\hline Liver & 16 & 13 & \multicolumn{2}{|l|}{11} & 40 \\
\hline All & 27 & 40 & \multicolumn{2}{|l|}{22} & \\
\hline \multicolumn{2}{|c|}{ KEGG pathway } & AMF versus $\mathrm{CO}$ & AMF-MFGM versus CO & AMF-M & AMF \\
\hline \multicolumn{6}{|l|}{ Muscle } \\
\hline \multicolumn{2}{|c|}{ Circadian rhythm (rno04710) } & $\sqrt{ }$ & $\sqrt{ }$ & $\sqrt{ }$ & \\
\hline \multicolumn{2}{|c|}{ PPAR signaling (rno03320) } & $\sqrt{ }$ & & $\sqrt{ }$ & \\
\hline \multicolumn{2}{|c|}{ Adipocytokine signaling (rno04920) } & & $\sqrt{ }$ & $\sqrt{ }$ & \\
\hline \multicolumn{6}{|l|}{ Adipose } \\
\hline \multicolumn{2}{|c|}{ Circadian rhythm (rno04710) } & $\sqrt{ }$ & $\sqrt{ }$ & \multicolumn{2}{|l|}{$\sqrt{ }$} \\
\hline \multicolumn{2}{|c|}{ Endocytosis (rno04710) } & $\sqrt{ }$ & $\sqrt{ }$ & \multicolumn{2}{|l|}{$\sqrt{ }$} \\
\hline \multicolumn{2}{|c|}{ Butanoate metabolism (rno00650) } & $\sqrt{ }$ & $\sqrt{ }$ & \multicolumn{2}{|l|}{$\sqrt{ }$} \\
\hline \multicolumn{2}{|c|}{ Citrate cycle (TCA cycle) (rno00020) } & $\sqrt{ }$ & $\sqrt{ }$ & & \\
\hline Liver & & & & & \\
\hline PPAR s & 03320) & $\sqrt{ }$ & $\sqrt{ }$ & $\sqrt{ }$ & \\
\hline C21 ste & metabolism (rno00140) & $\sqrt{ }$ & $\sqrt{ }$ & $\sqrt{ }$ & \\
\hline Biosynt & urated fatty acids (rno01040) & $\sqrt{ }$ & $\sqrt{ }$ & & \\
\hline Retinol & & $\sqrt{ }$ & $\sqrt{ }$ & & \\
\hline Starch a & netabolism (rno00500) & $\sqrt{ }$ & $\sqrt{ }$ & & \\
\hline Pyruvat & $(\operatorname{rno} 00620)$ & $\sqrt{ }$ & $\sqrt{ }$ & & \\
\hline Galacto & n (rno00052) & $\sqrt{ }$ & $\sqrt{ }$ & & \\
\hline Insulin & away (rno04910) & $\sqrt{ }$ & $\sqrt{ }$ & & \\
\hline Maturity & tes of the young (rno04950) & $\sqrt{ }$ & $\sqrt{ }$ & & \\
\hline Androg & en metabolism (rno00150) & $\sqrt{ }$ & $\sqrt{ }$ & & \\
\hline Glycero & metabolism (rno00564) & & $\sqrt{ }$ & $\sqrt{ }$ & \\
\hline
\end{tabular}

$C O$ corn oil, $A M F$ anhydrous milk fat, $A M F-M F G M$ anhydrous milk fat-milk fat globule membrane

Table 2 Differentially expressed genes by ANOVA in liver from rats fed with diet containing corn oil (control), anhydrous milk fat (AMF), and milk fat globule membrane (AMF-MFGM) (unit: $\Delta \Delta C(t)$ )

\begin{tabular}{lrrr}
\hline Genes & \multicolumn{1}{l}{ CO } & \multicolumn{1}{l}{ AMF } & \multicolumn{1}{c}{ MFGM } \\
\hline Cyp8b1 & $-0.94 \pm 0.75^{\mathrm{b}}$ & $-0.10 \pm 0.50^{\mathrm{a}}$ & $-2.49 \pm 0.93^{\mathrm{c}}$ \\
Agpat2 & $1.93 \pm 2.36^{\mathrm{ab}}$ & $2.82 \pm 1.14^{\mathrm{a}}$ & $1.14 \pm 1.03^{\mathrm{b}}$ \\
Angpt14 & $2.90 \pm 2.10^{\mathrm{b}}$ & $4.19 \pm 0.99^{\mathrm{a}}$ & $2.34 \pm 1.36^{\mathrm{b}}$
\end{tabular}

${ }^{a-c}$ Means in a row with different superscripts are significantly different $(p<0.05)$

determine potential cancer protective effects of specific lipids associated with this material, such as sphingomyelin and plasmalogens. The AMF diet was included to control
Table 3 Percentage of 20:3n-9 in highly unsaturated fatty acids (HUFA) in RBC, skeletal muscle, adipose, and liver as a function of diet

\begin{tabular}{lllr}
\hline \%20:3n-9 in HUFA & Corn oil & AMF & AMF-MFGM \\
\hline RBC & ND $^{\mathrm{b}}$ & $11.05 \pm 0.26^{\mathrm{a}}$ & $8.60 \pm 2.40^{\mathrm{a}}$ \\
Muscle & $0.51 \pm 0.08^{\mathrm{c}}$ & $20.01 \pm 0.45^{\mathrm{a}}$ & $12.44 \pm 0.28^{\mathrm{b}}$ \\
Adipose & $7.92 \pm 0.41^{\mathrm{c}}$ & $43.08 \pm 2.37^{\mathrm{a}}$ & $31.40 \pm 0.37^{\mathrm{b}}$ \\
Liver & $0.53 \pm 0.05^{\mathrm{c}}$ & $15.65 \pm 1.67^{\mathrm{a}}$ & $8.64 \pm 1.15^{\mathrm{b}}$ \\
\hline
\end{tabular}

$N D$ not detected

${ }^{\mathrm{a}-\mathrm{c}}$ Means in a row with different superscripts are significantly different $(p<0.05)$

for the potential for other lipids in milk fat to affect the development of ACF, such as butyrate and conjugated linoleic acid. 
Fig. 7 Hepatic Oil Red staining (a) and hepatic DGAT activity (b) in liver tissue from rats fed $\mathrm{CO}$ (corn oil), AMF (anhydrous milk fat), and AMF-MFGM (anhydrous milk fat-milk fat globule membrane). Means with different superscripts are significantly different $(p<0.05)$
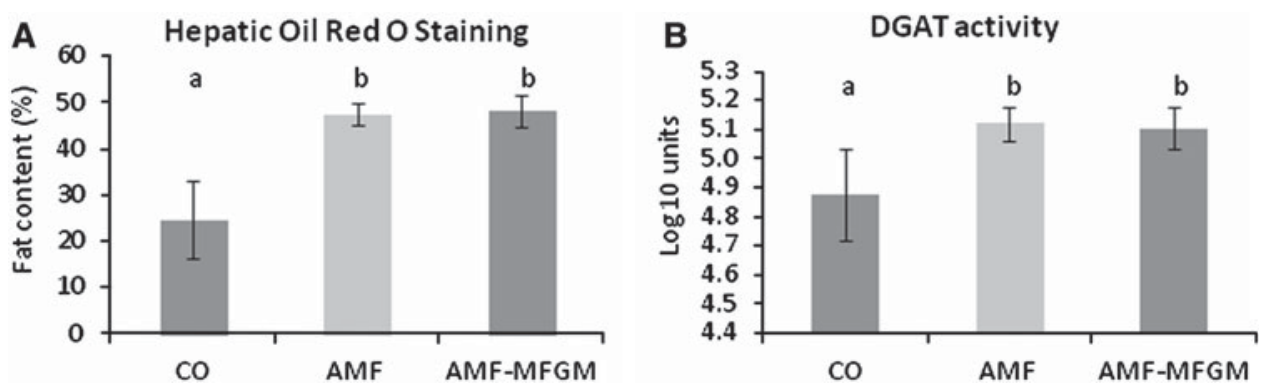

The membrane composition of RBC reflects dietary fat sources, and several groups have investigated the correlation of RBC fatty acid profiles with disease susceptibility in humans. For example, Harris and Von Schacky [26] showed that the omega-3 index, the combined percentage of EPA (20:5n-3) and DHA (22:6n-3) in RBC, correlates with the risk of coronary heart disease (CHD) in humans with values above $8 \%$ being associated with the most protection and values below $4 \%$ with the least. A similar metric, the percent contribution of omega- 6 fatty acids to highly unsaturated fatty acids (\% n-6 HUFA), was shown by Lands [27] to correlate with CHD mortality in several human populations with levels below $40 \%$ being protective. The omega- 3 indices were 0.9 for the $\mathrm{CO}$ diet, 2.5 for the AMF diet, and 2.0 for the AMF-MFGM diet, whereas the \% n-6 HUFA levels were 96,79 , and $83 \%$, respectively. Although these metrics may not be applicable in rodents, it is interesting nonetheless that all three diets result in very low levels of $n-3$ long chain PUFA in RBC which likely affect many physiological processes.

One interesting fatty acid which is present at significant levels in the RBC, muscle, adipose, and liver of the milkfat-fed animals, but not those consuming CO, is 20:3n-9. This fatty acid is an elongation product of oleic acid and has been shown to be a sensitive marker of PUFA intake. According to Lands [27], 20:3n-9 correlates with low PUFA intakes and a value of approximately $10 \%$ in the HUFA indicates PUFA insufficiency. To our knowledge, no studies have provided data on the percentage contribution of 20:3n-9 to tissue HUFA for RBC, muscle, adipose, and liver which are shown in Table 3. Animals fed the AMF diet have the highest levels of this fatty acid across all tissues measured, whereas those consuming the AMFMFGM diets also had high levels. Interestingly, the HUFA percentage of 20:3n-9 in adipose was high for the CO-fed animals, albeit not as high as the other groups. This may be a result of the very low concentration of HUFA in adipose, as the lipids in this tissue are primarily less than 18 carbons in length.

The lipid profiles of the RBC, muscle, adipose, and liver reflect the fat sources of the three diets, and there were few differences between those fed the AMF and AMF-MFGM.
However, there were quantitative differences across lipid classes in both plasma and the liver. Pathway analysis of the gene expression data indicated that the fewest pathways were affected in skeletal muscle and the most in liver. There were no differences in total liver fatty acids between treatments, but there was in the distribution of fatty acid across lipid classes (Fig. 6). One striking feature is the high FFA and low TAG in the livers of the CO-fed rats compared to those fed the AMF-MFGM diet. To further investigate this finding we compared Oil Red $\mathrm{O}$ staining in livers across the three treatments, and the results are shown in Fig. 7a. In the rats fed the $\mathrm{CO}$ diets there is less Oil Red O staining. According to O'Rourke et al. [28] Oil Red O staining of tissues is well correlated with triglyceride content. The inverse relationship between the hepatic TAG and FFA suggests that the differences may be due to triglyceride synthesis activity. According to the gene expression profiling, DGAT2, the enzyme responsible for the final step in TAG synthesis from diacylglycerols and FFA was 1.25 -fold lower in the CO-fed rats compared to those fed AMF and 1.46-fold lower compared to those fed AMF-MFGM. These differences are modest, and it was not clear if their expression would translate into physiological differences. Thus, the triacylglycerol synthetic activity in liver microsomal fractions was measured using a functional assay and the results are shown in Fig. 7b. In agreement with the Oil Red $\mathrm{O}$ staining, the DAG activity measure suggests that the differences in FFA, DAG, and TAG levels in the liver may be partially explained by the conversion of FFA and DAG into TAG.

Although excess hepatic TAG storage has typically been viewed as a negative physiological response, it may be protective under some metabolic situations. For example, in a mouse model of nonalcoholic steatohepatitis (NASH), Yamaguchi et al. [29] evaluated the effects of hepatic DGAT2 expression on disease development using an antisense nucleotide treatment. Although reducing expression of DGAT2 did prevent hepatic steatosis, the increase in FFA was associated with markers of lipid peroxidation/ oxidant stress and fibrosis. From this study the authors concluded that accumulation of hepatic TAG is actually protective against progressive liver damage in NAFLD. It 
is unclear why the $\mathrm{CO}$ diet resulted in lower DGAT expression and activity in the $\mathrm{CO}$-fed rats. However, one distinguishing feature of this diet is the PUFA content primarily in the form of linoleic acid. Interestingly, reviewing the fatty acid composition of diets used to study NASH, Romestaing et al. [30] drew the conclusion that dietary PUFA with a high omega-6 to omega-3 ratio may be a causative factor via effects on lipid peroxidation and proinflammatory cytokine production.

There are several differences in the plasma and hepatic lipid profiles of the animals fed the two milk fat diets which is surprising considering the similarity of these diets at the fatty acid level. According to the KEGG pathway analysis conducted on the hepatic gene expression and shown in Table 1, the two milk fat diets affected PPAR signaling (rno03320), C21 steroid hormone metabolism (rno00140), and glycerophospholipid metabolism (rno00564). The affected PPAR signaling in both the liver and skeletal muscle was mediated through $\operatorname{PPAR} \alpha$ receptors. The involved genes were associated with lipogenesis, cholesterol metabolism, and fatty acid transport in the liver and with lipogenesis in skeletal muscle. Consequently, the expression of three genes from these pathways was validated using RT-PCR. The genes Cyp8b1, Agpat2, and Angptl4 were selected from the differentially expressed genes in the pathways determined by the gene expression profiling. They were chosen owing to their physiological function and potential to affect tissue lipid partitioning, and the results are shown in Table 2. The function of these genes is discussed below including their potential roles in affecting lipid metabolism across the two milk fat groups. Of course these findings are only correlations and positive confirmation of their roles will need to be tested explicitly for confirmation.

Cyp8b1 is a cytochrome $\mathrm{P} 450$ enzyme which is a component of the primary bile acid biosynthesis and $\operatorname{PPAR} \alpha$ signaling pathways in the liver. Cyp8b1 is involved in the synthesis of cholic acid [31]. Cyp8b1 controls the ratio of cholic acid to chenodeoxycholic acid in bile salts, and as cholic acid is less effective in solubilizing cholesterol it may affect its absorption [32]. The AMF-MFGM diet was associated with a lower hepatic cholesteryl ester content (Fig. 6). Milk polar lipids affect both the absorption and fecal excretion of cholesterol when fed both acutely and chronically. For example, Noh and Koo [33] found that infusion of milk sphigomyelin via a duodenal catheter resulted in decreased cholesterol, fat, and vitamin E absorption. In a chronic feeding study, Kamili et al. [34] fed mice high-fat diets for 5 weeks; the diets were supplemented with two different formulations of milk phospholipids at $1.2 \%$ by weight. Both milk polar lipid preparations resulted in significant decreases in liver cholesterol and triglyceride which was accompanied by an increase in fecal cholesterol excretion. Data from the present study indicate that the decreased cholesterol absorption and increased fecal excretion resulting from luminal milk polar lipids in those previous studies may be mediated, at least in part, by the ratio of bile acids.

Agpat2 is an enzyme responsible for the conversion of lysophosphatidic acid to phosphatidic acid, a step in PL synthesis, and is a component of KEGG pathway glycerophospholipid metabolism. The expression of Agpat2 was higher in the AMF-MFGM group than the AMF group (Table 2) but there was not a significant difference in hepatic PL. There was, on the other hand, a significant increase in plasma PL in the AMF-MFGM group. The increased plasma PL may result from increased hepatic synthesis and secretion. Previous studies with milk polar lipids have also shown the ability of these constituents to increase both plasma PL and TAG. For example, Wat et al. [35] fed mice both a low-fat, non-purified diet and a highfat diet formulated with and without a $2.5 \%$ PL-rich milk fat extract. In the mice fed the low-fat, non-purified diet, the milk PL increased the plasma TAG by $20 \%$ and the plasma PL by $5 \%$ compared with values in the control mice fed the low-fat diet. However, in the mice fed the high-fat diets, the PL-rich extract actually reduced both the plasma TAG and PL to levels similar to the low-fat control. The increased plasma lipid concentration in the rats fed the AMF-MFGM diet, compared to those fed the AMF diet, may be a result of either more lipid exported from the liver, or from reduced clearance via peripheral tissues.

Expression of Angptl4 was also verified by RT-PCR. Angptl4 is a component of the PPAR $\alpha$ signaling pathway. It is an angiopoietin-like protein which increases plasma TAG via suppression of lipoprotein lipase [36]. Interestingly, there were significant differences in plasma TAG between the two milk-fat-fed groups (Fig. 6). Also known as fasting-induced adipocyte factor (Fiaf), Angptl4 is highly expressed in the gut epithelium in germ-free mice and prevents accumulation of fat into adipocytes, whereas conventionalization with a microflora suppresses this effect [37]. Although it is not clear if hepatic expression of Angptl4 in the AMF-MFGM-fed rats contributed to the increase in plasma TAG, this finding indicates a potential mechanism via which this effect was mediated.

Despite the fact that it is still commonly used in ACF studies with rodents [7], the high sucrose content of the AIN-76A diet has been long known to cause fatty liver in animals fed the diet for long periods of time [9]. Therefore, when reviewing the different effects of the fat sources on tissue lipid composition and gene expression it is necessary to keep in mind that these changes are in the context of an overall metabolically stressful diet. From the data presented here, a few hypotheses may be drawn that can be tested in studies designed specifically for their evaluation. 
For example, compared to the milk fat diets, the $\mathrm{CO}$ diet caused a significant increase in hepatic free fatty acids, a potential trigger for the development of NASH from NAFLD. Conversely, compared to the CO and AMF-MFGM diets, the AMF diet resulted in more hepatic TAG storage which in itself may be undesirable. Supplementing the AMF diet with polar lipids from milk appears to reduce the accumulation of hepatic TAG yet appears to achieve this effect via promotion of lipid export into plasma. The long-term physiological significance of this is unknown. In conclusion, the fat source of the AIN-76A diet affects the tissue profile of key tissues involved in lipid trafficking and storage as well as gene expression networks within these tissues.

Acknowledgments This study was supported by both a seed grant from the Center for Integrated Biosystems at Utah State University and by the Utah Agricultural Experiment Station and was approved as journal paper number 8290. We are grateful to Dallin Snow for preparing RNA samples for Microarray analysis and Dr. Aaron Olsen and Kent Udy for assistance with animal studies.

\section{References}

1. Reeves PG, Nielsen FH, Fahey GC Jr (1993) AIN-93 purified diets for laboratory rodents: final report of the American Institute of Nutrition ad hoc writing committee on the reformulation of the AIN-76A rodent diet. J Nutr 123(11):1939-1951

2. Roebuck BD, Wilpone SA, Fifield DS, Yager JD Jr (1979) Hemorrhagic deaths with AIN-76 diet. J Nutr 109(5):924-925

3. Nguyen HT, Woodard JC (1980) Intranephronic calculosis in rats: an ultrastructural study. Am J Pathol 100(1):39-56

4. Medinsky MA, Popp JA, Hamm TE, Dent JG (1982) Development of hepatic lesions in male Fischer-344 rats fed AIN-76A purified diet. Toxicol Appl Pharmacol 62(1):111-120

5. Nutrition A (1980) Second report of the ad hoc committee on standards for nutritional studies. J Nutr 110:1726

6. Council NR (1995) Nutrient requirements of laboratory animals, vol 4th Revised. National Academy Press, Washington, p 172

7. Corpet DE, Tache S (2002) Most effective colon cancer chemopreventive agents in rats: a systematic review of aberrant crypt foci and tumor data, ranked by potency. Nutr Cancer 43(1):1-21

8. Targher G, Day CP, Bonora E (2010) Risk of cardiovascular disease in patients with nonalcoholic fatty liver disease. $\mathrm{N}$ Engl $\mathrm{J}$ Med 363(14):1341-1350

9. Bacon BR, Park CH, Fowell EM, McLaren CE (1984) Hepatic steatosis in rats fed diets with varying concentrations of sucrose. Fundam Appl Toxicol 4(5):819-826

10. Snow DR, Jimenez-Flores R, Ward RE, Cambell J, Young MJ, Nemere I, Hintze KJ (2010) Dietary milk fat globule membrane reduces the incidence of aberrant crypt foci in Fischer-344 rats. J Agric Food Chem 58(4):2157-2163

11. Jensen RG (1995) Handbook of milk composition: food science and technology international series. Academic, San Diego, p 919

12. Mcpherson AV, Dash MC, Kitchen BJ (1984) Isolation of bovinemilk fat globule-membrane material from cream without prior removal of caseins and whey proteins. J Dairy Res 51(1):113-121

13. MacGibbon A, Taylor M (2006) Composition and structure of bovine milk lipids. In: Fox PF, McSweeney PLH (eds) Advanced dairy chemistry. Springer, New York, pp 1-42

14. Spitsberg VL (2005) Invited review: bovine milk fat globule membrane as a potential nutraceutical. J Dairy Sci 88:2289-2294
15. Folch J, Lees M, Sloane Stanley GH (1957) A simple method for the isolation and purification of total lipides from animal tissues. J Biol Chem 226(1):497-509

16. Curtis JM, Berrigan N, Dauphinee P (2008) The determination of n-3 fatty acid levels in food products containing microencapsulated fish oil using the one-step extraction method. Part 1: measurement in the raw ingredient and in dry powdered foods. J Am Oil Chem Soc 85(4):297-305

17. Blazejczyk M, Miron M, Nadon R (2007) FlexArray: a statistical data analysis software for gene expression microarrays. http://genomequebec.mcgill.ca/FlexArray. Accessed 8 Oct 2012

18. Long AD, Mangalam HJ, Chan BY, Tolleri L, Hatfield GW, Baldi P (2001) Improved statistical inference from DNA microarray data using analysis of variance and a Bayesian statistical framework: analysis of global gene expression in Escherichia coli K12. J Biol Chem 276(23):19937-19944

19. Hochberg Y, Benjamini Y (1990) More powerful procedures for multiple significance testing. Stat Med 9(7):811-818

20. Tong W, Cao X, Harris S, Sun H, Fang H, Fuscoe J, Harris A, Hong H, Xie Q, Perkins R, Shi L, Casciano D (2003) ArrayTrack-supporting toxicogenomic research at the US Food and Drug Administration National Center for Toxicological Research. Environ Health Perspect 111(15):1819-1826

21. Lopez-Parra M, Titos E, Horrillo R, Ferre N, Gonzalez-Periz A, Martinez-Clemente M, Planaguma A, Masferrer J, Arroyo V, Claria J (2008) Regulatory effects of arachidonate 5-lipoxygenase on hepatic microsomal TG transfer protein activity and VLDL-triglyceride and apoB secretion in obese mice. J Lipid Res 49(12):2513-2523

22. Abramoff MD, Magalhaes PJ, Ram SJ (2004) Image processing with ImageJ. Biophotonics Int 11(7):36-42

23. Ko JS, Ryu SY, Kim YS, Chung MY, Kang JS, Rho MC, Lee HS, Kim YK (2002) Inhibitory activity of diacylglycerol acyltransferase by tanshinones from the root of Salvia miltiorrhiza. Arch Pharm Res 25(4):446-448

24. Coleman RA (1992) Diacylglycerol acyltransferase and monoacylglycerol acyltransferase from liver and intestine. Methods Enzymol 209:98-104

25. McFie PJ, Stone SJ (2011) A fluorescent assay to quantitatively measure in vitro acyl CoA:diacylglycerol acyltransferase activity. J Lipid Res 52(9):1760-1764

26. Harris WS, Von Schacky C (2004) The omega-3 index: a new risk factor for death from coronary heart disease? Prev Med 39(1):212-220

27. Lands B (2008) A critique of paradoxes in current advice on dietary lipids. Prog Lipid Res 47(2):77-106

28. O'Rourke EJ, Soukas AA, Carr CE, Ruvkun G (2009) C. elegans major fats are stored in vesicles distinct from lysosome-related organelles. Cell Metab 10(5):430-435

29. Yamaguchi K, Yang L, McCall S, Huang J, Yu XX, Pandey SK, Bhanot S, Monia BP, Li YX, Diehl AM (2007) Inhibiting triglyceride synthesis improves hepatic steatosis but exacerbates liver damage and fibrosis in obese mice with nonalcoholic steatohepatitis. Hepatology 45(6):1366-1374

30. Romestaing C, Piquet MA, Bedu E, Rouleau V, Dautresme M, Hourmand-Ollivier I, Filippi C, Duchamp C, Sibille B (2007) Long term highly saturated fat diet does not induce NASH in Wistar rats. Nutr Metab (Lond) 4:4

31. Li-Hawkins J, Gafvels M, Olin M, Lund EG, Andersson U, Schuster G, Bjorkhem I, Russell DW, Eggertsen G (2002) Cholic acid mediates negative feedback regulation of bile acid synthesis in mice. J Clin Invest 110(8):1191-1200

32. Gafvels M, Olin M, Chowdhary BP, Raudsepp T, Andersson U, Persson B, Jansson M, Bjorkhem I, Eggertsen G (1999) Structure and chromosomal assignment of the sterol 12alphahydroxylase gene (CYP8B1) in human and mouse: eukaryotic 
cytochrome P-450 gene devoid of introns. Genomics 56(2):184-196

33. Noh SK, Koo SI (2004) Milk sphingomyelin is more effective than egg sphingomyelin in inhibiting intestinal absorption of cholesterol and fat in rats. J Nutr 134(10):2611-2616

34. Kamili A, Wat E, Chung RW, Tandy S, Weir JM, Meikle PJ, Cohn JS (2010) Hepatic accumulation of intestinal cholesterol is decreased and fecal cholesterol excretion is increased in mice fed a high-fat diet supplemented with milk phospholipids. Nutr Metab (Lond) 7:90
35. Wat E, Tandy S, Kapera E, Kamili A, Chung RW, Brown A, Rowney M, Cohn JS (2009) Dietary phospholipid-rich dairy milk extract reduces hepatomegaly, hepatic steatosis and hyperlipidemia in mice fed a high-fat diet. Atherosclerosis 205(1):144-150

36. Kersten S (2005) Regulation of lipid metabolism via angiopoietin-like proteins. Biochem Soc Trans 33(Pt 5):1059-1062

37. Backhed F, Ding H, Wang T, Hooper LV, Koh GY, Nagy A, Semenkovich CF, Gordon JI (2004) The gut microbiota as an environmental factor that regulates fat storage. Proc Natl Acad Sci U S A 101(44):15718-15723 